TRANSACTIONS OF THE

AMERICAN MATHEMATICAL SOCIETY

Volume 353, Number 2, Pages 795-807

S 0002-9947(00)02750-

Article electronically published on October 23, 2000

\title{
STRICHARTZ ESTIMATES IN THE HYPERBOLIC SPACE AND GLOBAL EXISTENCE FOR THE SEMILINEAR WAVE EQUATION
}

\author{
DANIEL TATARU
}

\begin{abstract}
The aim of this article is twofold. First we consider the wave equation in the hyperbolic space $\mathbb{H}^{n}$ and obtain the counterparts of the Strichartz type estimates in this context. Next we examine the relationship between semilinear hyperbolic equations in the Minkowski space and in the hyperbolic space. This leads to a simple proof of the recent result of Georgiev, Lindblad and Sogge on global existence for solutions to semilinear hyperbolic problems with small data. Shifting the space-time Strichartz estimates from the hyperbolic space to the Minkowski space yields weighted Strichartz estimates in $\mathbb{R}^{n} \times \mathbb{R}$ which extend the ones of Georgiev, Lindblad, and Sogge.
\end{abstract}

\section{INTRODUCTION}

We start by explaining the relationship between the wave equation in $\mathbb{R}^{n} \times \mathbb{R}$ and the wave equation in the hyperbolic space. Denote by $(x, t)$ the cartesian coordinates in the Minkowski space $\mathbb{R}^{n} \times \mathbb{R}$ and by $(r, \Theta)$ the polar coordinates in $\mathbb{R}^{n}, x=r \Theta, r=|x|$. In the forward light cone $\left\{t^{2}-x^{2}>0, t>0\right\}$ introduce the new coordinates $(\tau, s, \Theta)$ by

$$
\begin{aligned}
x & =\Theta e^{\tau} \sinh s, \\
t & =e^{\tau} \cosh s .
\end{aligned}
$$

We interpret $(s, \Theta)$ as the polar coordinates in the hyperbolic space $\mathbb{H}^{n}$, while $\tau$ will be the substitute for time. The level surfaces $\tau=$ const are the hyperboloids

$$
t^{2}-x^{2}=e^{2 \tau} .
$$

The volume element changes to

$$
d x d t=r^{n-1} d x d r d \Theta=e^{(n+1) \tau}(\sinh s)^{n-1} d \tau d s d \Theta,
$$

which further gives

$$
d x d t=e^{(n+1) \tau} d \tau \omega,
$$

where $\omega$ is the volume element in the hyperbolic space $\mathbb{H}^{n}$.

Denote by $\square$ the wave operator in the Minkowski space,

$$
\square=-\partial_{t}^{2}+\Delta_{x} .
$$

Received by the editors October 10, 1997.

2000 Mathematics Subject Classification. Primary 35L05, 35L70; Secondary 58J45.

Key words and phrases. Semilinear wave equation, Strichartz estimates, hyperbolic space.

Research partially supported by NSF grant DMS-9622942 and by an Alfred P. Sloan fellowship. 
In polar coordinates it can be expressed as

$$
\square=-\partial_{t}^{2}+\partial_{r}^{2}+\frac{n-1}{r} \partial_{r}+\frac{1}{r^{2}} \partial_{\Theta}^{2},
$$

where $\partial_{\Theta}^{2}$ is the spherical Laplacian. Now we want to write $\square$ in the new coordinates. Set

$$
\rho=\frac{n-1}{2} .
$$

Then a simple computation leads to

$$
\square=e^{-2 \tau}\left(-\partial_{\tau}^{2}-2 \rho \partial_{\tau}+\Delta_{H}\right),
$$

where $\Delta_{H}$ is the Laplace-Beltrami operator in the hyperbolic space, whose expression in polar coordinates is

$$
\Delta_{H}=\partial_{s}^{2}+2 \rho(\operatorname{coth} s) \partial_{s}+(\sinh s)^{-2} \partial_{\Theta}^{2} .
$$

This expression can be simplified if we conjugate it by $e^{\rho \tau}$. Then we obtain

$$
e^{\rho \tau} \square e^{-\rho \tau}=e^{-2 \tau} \square_{H},
$$

where $\square_{H}$ is the wave operator in the hyperbolic space,

$$
\square_{H}=-\partial_{\tau}^{2}+\left(\Delta_{H}+\rho^{2}\right)
$$

For the convenience of the reader we recall that the spectrum of $-\Delta_{H}$ is $\left[\rho^{2}, \infty\right)$.

\section{THE SPHERICAL FUNCTION AND THE SPHERICAL TRANSFORM IN $\mathbb{H}^{n}$}

The aim of this section is to give a brief overview of the spherical function and the spherical Fourier transform in $\mathbb{H}^{n}$. We direct the reader who is interested in more details to [2] and the references therein. In the more general context of symmetric spaces, see [10].

Given a spherically symmetric function $f=f(s)$ in $\mathbb{H}^{n}$, we can define its spherical transform (which is an analogue of the Fourier transform in $\mathbb{R}^{n}$ acting on spherically symmetric functions) by

$$
\tilde{f}(\lambda)=\int_{0}^{\infty} f(s) \Phi_{\lambda}(s)(\sinh s)^{2 \rho} d s, \quad \lambda \in[0, \infty),
$$

where $\Phi_{\lambda}(s)$ is the spherical function in $\mathbb{H}^{n}$. Of the many integral formulas for $\Phi_{\lambda}(s)$ we shall use the following (see [2]):

$$
\Phi_{\lambda}(s)=\frac{2^{\rho-1} \Gamma\left(\rho+\frac{1}{2}\right)}{\Gamma(\rho)(\sinh s)^{2 \rho-1}} \int_{-s}^{s} e^{i \lambda \mu}(\cosh s-\cosh \mu)^{\rho-1} d \mu .
$$

Observe that, as a function of $\lambda, \Phi_{\lambda}(s)$ can be extended to an even entire function in the complex plane.

The inversion formula (an "inverse Fourier transform") is

$$
f(s)=\int_{0}^{\infty} \tilde{f}(\lambda) \Phi_{\lambda}(s)|c(\lambda)|^{-2} d \lambda,
$$

where $c(\lambda)$ is the Harish-Chandra c-function,

$$
c(\lambda)=\frac{2^{2 \rho-1} \Gamma\left(\rho+\frac{1}{2}\right) \Gamma(i \lambda)}{\pi^{1 / 2} \Gamma(\rho+i \lambda)} .
$$


Spherically symmetric multipliers can be defined in an abstract fashion in $\mathbb{H}^{n}$ as functions of the modified Laplace-Beltrami operator $-\Delta_{H}-\rho^{2}$. Given a symbol $a(\lambda)$, the inversion formula yields the spherically symmetric kernel of the corresponding operator $A(|D|),|D|=\left(-\Delta_{H}-\rho^{2}\right)^{\frac{1}{2}}$, as

$$
K(s)=\int_{0}^{\infty} a(\lambda) \Phi_{\lambda}(s)|c(\lambda)|^{-2} d \lambda,
$$

where

$$
(A f)(z)=\int_{H^{n}} f(y) K([z, y]) d y .
$$

Here $[z, y]$ denotes the Riemannian distance between $y$ and $z$ in $\mathbb{H}^{n}$.

As usual, all multipliers with bounded symbols are $L^{2}$ bounded. The question of the $L^{p}$ boundedness of spherically symmetric multipliers has been addressed in 4]. Unlike in $\mathbb{R}^{n}$, here one needs symbols which have a holomorphic extension in a strip if $p \neq 2$.

Theorem 1. Let $1<p<\infty$ and $\beta(p)=\rho\left|1-\frac{2}{p}\right|$. Let $a(\lambda)$ be an even holomorphic $S^{0}$ symbol in the strip $|\Im \lambda| \leq \beta(p)$. Then the multiplier $A(|D|)$ is bounded in $L^{p}\left(\mathbb{H}^{n}\right)$.

We conclude this section with some precise asymptotics at infinity for $c^{-1}(\lambda)$ and $\Phi_{\lambda}(s)$, which are needed in the sequel. For $c^{-1}(\lambda)$ we have the following lemma:

Lemma 2.1. The Harish-Chandra $c$-function $c(\lambda)$ has the following properties:

a) $c^{-1}(\lambda)$ is a holomorphic $S^{\rho}$ symbol in the strip $|\Im \lambda| \leq \rho$ with the possible exception of a simple pole at $\lambda=-i \rho$.

b) The principal symbol of $c^{-1}$ is $\frac{2^{2 \rho-1} \Gamma\left(\rho+\frac{1}{2}\right)}{\pi^{1 / 2}}(i \lambda+0)^{\rho}$.

This follows directly from (8). Observe that (b) implies that for large $\lambda$ we have

$$
c^{-1}(\lambda)=\frac{2^{2 \rho-1} \Gamma\left(\rho+\frac{1}{2}\right)}{\pi^{1 / 2}}(i \lambda+0)^{\rho}+O\left(|\lambda|^{\rho-1}\right) .
$$

For the spherical function $\Phi_{\lambda}(s)$, matters are a bit more complicated. Start with the following bound, which is a simple consequence of ([6):

$$
\left|\Phi_{\lambda}(s)\right| \leq c e^{-\rho s}(1+s), \quad \lambda \in \mathbb{R} .
$$

This is good enough for small $\lambda$ but is imprecise as $\lambda \rightarrow \infty$. A better result is

Lemma 2.2. a) Suppose that $s \geq 1$. Then

$$
(\sinh s)^{\rho} \Phi_{\lambda}(s)=2^{\rho} \Gamma\left(\rho+\frac{1}{2}\right) \cos \left(|\lambda| s-\frac{\rho \pi}{2}\right)|\lambda|^{-\rho}+O\left(|\lambda|^{-\rho-1}\right), \quad \lambda \geq 1,
$$

and

$$
(\sinh s)^{\rho} \Phi_{\lambda}(s)=\frac{2^{\rho} \Gamma\left(\rho+\frac{1}{2}\right)}{\Gamma(\rho)} \frac{\sin \lambda s}{\lambda}+O(1), \quad 0 \leq \lambda \leq 1 .
$$

b) Suppose that $s \leq 1, s \lambda \geq 1$. Then

$$
(\sinh s)^{\rho} \Phi_{\lambda}(s)=2^{\rho} \Gamma\left(\rho+\frac{1}{2}\right) \cos \left(|\lambda| s-\frac{\rho \pi}{2}\right)|\lambda|^{-\rho}+O\left(s^{-1}|\lambda|^{-\rho-1}\right) .
$$


Proof. (a1) The estimate for large $s$ and large $\lambda$. We use the formula (6). First we cut off the integral at $\pm(s-1)$. Thus, let $\chi$ be a smooth cutoff function so that $\chi(x)=1$ for $x<\frac{1}{2}$ and $\chi(x)=0$ for $x \geq 1$. Then we claim that the integral

$$
I=(\sinh s)^{1-2 \rho} \int_{-s}^{s} e^{i \lambda \mu}(1-\chi(s-|\mu|))(\cosh s-\cosh \mu)^{\rho-1} d \mu
$$

is rapidly decaying as $\lambda$ approaches infinity. Indeed, we have

$$
\partial_{\mu}^{\alpha}\left[(1-\chi(s-|\mu|))(\cosh s-\cosh \mu)^{\rho-1}\right] \leq c_{\alpha} \cosh \mu(\cosh s)^{\rho-2}, \quad \alpha \geq 1 ;
$$

therefore for $I$ we obtain

$$
|I| \leq c_{N} e^{-\rho s}|\lambda|^{-N}, \quad N \geq 1 .
$$

Hence, to prove (12) we still need to estimate

$$
\begin{aligned}
J & =(\sinh s)^{1-2 \rho} \int_{-s}^{s} e^{i \lambda \mu} \chi(s-|\mu|)(\cosh s-\cosh \mu)^{\rho-1} d \mu \\
& =2(\sinh s)^{1-2 \rho \Re} \int_{s-1}^{s} e^{i \lambda \mu} \chi(s-\mu)(\cosh s-\cosh \mu)^{\rho-1} d \mu .
\end{aligned}
$$

Use the change of variable $\mu:=s-\mu$ to rewrite $J$ as

$$
\begin{aligned}
J & =2(\sinh s)^{1-2 \rho \Re e^{i \lambda s}} \int_{0}^{1} e^{-i \lambda \mu} \chi(\mu)(\cosh s-\cosh (s-\mu))^{\rho-1} d \mu \\
& =2(\sinh s)^{1-2 \rho \Re e^{i \lambda s}} \int_{0}^{1} e^{-i \lambda \mu} \chi(\mu)\left(2 \sinh \left(s-\frac{\mu}{2}\right) \sinh \frac{\mu}{2}\right)^{\rho-1} d \mu \\
& =2(\sinh s)^{-\rho} \Re e^{i \lambda s}\left(\int_{0}^{1} e^{-i \lambda \mu} \chi(\mu) \mu^{\rho-1} d \mu+\int_{0}^{1} e^{-i \lambda \mu} g(s, \mu) \mu^{\rho} d \mu\right),
\end{aligned}
$$

where

$$
g(s, \mu)=\chi(\mu) \mu^{-1}\left[\left(\frac{2 \sinh \frac{\mu}{2} \sinh \left(s-\frac{\mu}{2}\right)}{\mu \sinh s}\right)^{\rho-1}-1\right]
$$

is smooth and bounded, uniformly in $s>1, \mu \in \mathbb{R}$. Now for large $\lambda$ the first integral equals $\Gamma(\rho)(-i \lambda+0)^{-\rho}$ modulo a rapidly decaying component, while the second integral has an additional factor of $\mu$ and therefore decays one order better, i.e. like $|\lambda|^{-\rho-1}$. Hence, for large $\lambda$ we have

$$
J=2 \Gamma(\rho)(\sinh s)^{-\rho}\left(\Re e^{i \lambda s}(-i \lambda+0)^{-\rho}+O\left(|\lambda|^{-\rho-1}\right)\right) .
$$

Adding $I$ and $J$, by (6) we get (12). q.e.d.

(a2) The estimate for large $s$, small $\lambda$. We replace $(\cosh s-\cosh \mu)^{\rho-1}$ by $(\sinh s)^{\rho-1}$ in the integrand in (6) and estimate the error. This yields

$$
\begin{aligned}
\int_{-s}^{s} e^{i \lambda \mu}(\cosh s-\cosh \mu)^{\rho-1} d \mu & =(\sinh s)^{\rho-1} \int_{-s}^{s} e^{i \lambda \mu} d \mu+O\left((\sinh s)^{\rho-1}\right) \\
& =2(\sinh s)^{\rho-1} \frac{\sin \lambda s}{\lambda}+O\left((\sinh s)^{\rho-1}\right),
\end{aligned}
$$

which implies (13).

(b) The estimate for small $s$. Again, we are looking at

$$
(\sinh s)^{\rho} \Phi_{s}(\lambda)=\frac{2^{\rho-1} \Gamma\left(\rho+\frac{1}{2}\right)}{\Gamma(\rho)} \int_{-s}^{s} e^{i \lambda \mu} a(s, \mu) d \mu,
$$


where

$$
a(s, \mu)=\sinh s^{1-\rho}(\cosh s-\cosh \mu)^{\rho-1} .
$$

We claim that we have the representation

$$
a(s, \mu)=(2 s)^{1-\rho}\left(\left(s^{2}-\mu^{2}\right)^{\rho-1}+g(s, \mu)\left(s^{2}-\mu^{2}\right)^{\rho}\right),
$$

where $g$ is a smooth function. Indeed,

$$
\begin{aligned}
a(s, \mu) & =(\sinh s)^{1-\rho}\left(2 \sinh \frac{s+\mu}{2} \sinh \frac{s-\mu}{2}\right)^{\rho-1} \\
& =(2 s)^{1-\rho}\left(s^{2}-\mu^{2}\right)^{\rho-1} b(s, \mu),
\end{aligned}
$$

where $b(s, \mu)$ is the smooth function

$$
b(s, \mu)=\left(\frac{2 s}{\sinh s} \frac{\sinh \frac{s+\mu}{2}}{\frac{s+\mu}{2}} \frac{\sinh \frac{s-\mu}{2}}{\frac{s-\mu}{2}}\right)^{\rho-1} .
$$

Since $b$ satisfies $b( \pm s, s)=1$, we can decompose it as

$$
b(s, \mu)=1+\left(s^{2}-\mu^{2}\right) g(s, \mu),
$$

where $g$ is a smooth function. This proves (16).

Next we estimate the principal part of the integral (15),

$$
\begin{aligned}
J & =\int_{-s}^{s} e^{i \lambda \mu}(2 s)^{1-\rho}\left(s^{2}-\mu^{2}\right)^{\rho-1} d \mu \\
& =\int_{-1}^{1} e^{i \lambda s \mu} 2^{1-\rho} s^{\rho}\left(1-\mu^{2}\right)^{\rho-1} d \mu .
\end{aligned}
$$

This gives

$$
J=2^{1-\rho} s^{\rho} d(\lambda s)
$$

where $d$ is the Fourier transform of $\left(1-\mu^{2}\right)_{+}^{\rho-1}$. Then $d$ has the form

$$
d(\xi)=2^{\rho} \Gamma(\rho) \Re e^{i \xi}(-i \xi+0)^{-\rho}+O\left(|\xi|^{-\rho-1}\right) .
$$

Hence, for $\lambda s>1$ we get

$$
J=2 \Gamma(\rho) s^{-\rho} \Re e^{i \lambda s}(-i \lambda+0)^{-\rho}+O\left(s^{-\rho-1}|\lambda|^{-\rho-1}\right) .
$$

The similar result with $\rho$ replaced by $\rho+1$ yields the bound for the remainder,

$$
I=\int_{-s}^{s} e^{i \lambda \mu} g(s, \mu)\left(s^{2}-\mu^{2}\right)^{\rho} d \mu=O\left((\lambda s)^{-\rho-1}\right) .
$$

Adding $I$ and $J$ yields (14). q.e.d.

\section{Inhomogeneous Sobolev Spaces in the hyperbolic SPACE $\mathbb{H}^{n}$}

The aim of this section is to define the inhomogeneous Sobolev spaces $H^{s, p}$ in $\mathbb{H}^{n}$. We start with a more intuitive definition and then relate it to multipliers in $\mathbb{H}^{n}$.

Observe first that the $H^{s, p}$ norms can be easily defined, as in $\mathbb{R}^{n}$, for functions supported in a ball of fixed radius, say 4. An invariant way to do that is to fix a point $O$, introduce the geodesic local coordinates in the ball of radius 4 in $\mathbb{H}^{n}$ centered at $O$, and define the $H_{O}^{s, p}$ norm of functions in $\mathbb{H}^{n}$ supported in $B(0,4)$ 
as the pull-back of the $H^{s, p}$ norm in $\mathbb{R}^{n}$ in the local coordinates. Then for another $z \in \mathbb{H}^{n}$ we can define the $H_{z}^{s, p}$ norm for functions supported in $B(z, 4)$ as

$$
\|f\|_{H_{z}^{s, p}}=\|f \circ g\|_{H_{O}^{s, p}}
$$

where $g$ is a Lorentz transformation so that $g(O)=z$.

It remains to derive a global definition from this. Choose a countable collection of points $x_{k} \in \mathbb{H}^{n}$ with the following properties:

a) $\bigcup_{k} B\left(x_{k}, 2\right)=\mathbb{H}^{n}$.

b) $d\left(x_{k}, x_{m}\right) \geq 1$.

Corresponding to these points, choose a smooth partition of unit $\phi_{k}$ in $\mathbb{H}^{n}$, uniformly bounded in $C^{\infty}$, so that $\phi_{k}(x)=0$ if $d\left(x, x_{k}\right) \geq 4$.

Now define the global spaces $H^{s, p}$ in terms of their local version,

$$
u \in H^{s, p} \quad \text { if } \sum\left\|\phi_{k} u\right\|_{H_{x_{k}}^{s, p}}^{p}<\infty
$$

with the corresponding norm. It is easy to see that the spaces defined like this are Lorentz invariant and do not depend on the choice of the points $x_{k}$.

These spaces have the usual duality and interpolation properties, which can be derived as in [1, Chapter 6 . Next we use multipliers to give another equivalent characterization for these spaces.

Theorem 2. Let $s \in \mathbb{R}, 1<p<\infty$. Then $u \in H^{s, p}$ iff $\Delta_{H}^{s / 2} u \in L^{p}$.

Proof. Observe first that $\Delta_{H}^{i y}$ has the symbol $\left(\lambda^{2}+\rho^{2}\right)^{i y}$, which is $S^{0}$ in the strip $|\Im \lambda|<\rho$; therefore, by Theorem 1 it is $L^{p}$ bounded. Then by duality and interpolation the problem reduces to the case when $s$ is a nonnegative even integer. Clearly

$$
\left\|\Delta_{H}^{k} u\right\|_{L^{p}} \leq c\|u\|_{H^{2 k, p}} .
$$

It remains to prove the converse inequality,

$$
\left\|\Delta_{H}^{k} u\right\|_{L^{p}} \geq c\|u\|_{H^{2 k, p}} .
$$

By Theorem 1, if $k$ is a nonnegative integer then $\Delta_{H}^{-k}$ is bounded in $L^{p}$. Therefore

$$
\|u\|_{L^{p}} \leq\left\|\Delta_{H}^{k} u\right\|_{L^{p}} .
$$

Then, according to the definition of the $H^{2 k, p}$ space, (19) would follow from the estimate

$$
\left\|\phi_{k} u\right\|_{H_{x_{k}}^{2 k, p}} \leq c\left(\|u\|_{L^{p}\left(B\left(x_{k}, 4\right)\right)}+\left\|\Delta_{H}^{k} u\right\|_{L^{p}\left(B\left(x_{k}, 4\right)\right)}\right) .
$$

But this follows from standard local elliptic theory. q.e.d.

\section{Strichartz estimates in the hyperboliC SPACE}

We recall first the fixed time estimates for the wave equation in $\mathbb{R}^{n+1}$, from which the space-time Strichartz estimates can be derived (see [3], 6], 15], [20]). Define the operator $A(t)$ by

$$
A(t) f=u(t),
$$

where $u$ is the unique solution for the equation

$$
\left\{\begin{array}{l}
\square u=0, \\
u(0)=0, \\
u_{t}(0)=f .
\end{array}\right.
$$


Then the following estimate holds:

$$
\|A(t) f\|_{H^{-s+1, p}} \leq t^{-\rho\left(\frac{1}{p^{\prime}}-\frac{1}{p}\right)}\|f\|_{H^{s}, p^{\prime}},
$$

where

$$
2 s=(\rho+1)\left(\frac{1}{p^{\prime}}-\frac{1}{p}\right), \quad 2 \leq p<\infty .
$$

Here $H^{s, p}$ are the homogeneous Sobolev spaces in $\mathbb{R}^{n}$.

Now we are interested in the counterpart of this estimate corresponding to the wave equation in the hyperbolic space $\mathbb{H}^{n}$. Thus, consider the operators

$$
S(\tau) f=v(\tau), \quad C(\tau) f=v_{\tau}(\tau),
$$

where $v$ is the unique solution for the hyperbolic equation in $\mathbb{R} \times \mathbb{H}^{n}$

$$
\left\{\begin{array}{l}
\square_{H} v=0, \\
v(0)=0, \\
v_{\tau}(0)=f .
\end{array}\right.
$$

Then we have the following estimates:

Theorem 3. Suppose that (22) holds. Then

$$
\|S(\tau) f\|_{H^{-s+1, p}} \leq \frac{(1+\tau)^{\frac{2}{p}}}{(\sinh \tau)^{\rho\left(\frac{1}{p^{\prime}}-\frac{1}{p}\right)}}\|f\|_{H^{s, p^{\prime}}}
$$

and

$$
\|C(\tau) f\|_{H^{-s, p}} \leq \frac{1}{(\sinh \tau)^{\rho\left(\frac{1}{p^{\prime}}-\frac{1}{p}\right)}}\|f\|_{H^{s, p^{\prime}}} .
$$

Proof. We prove only (23); the proof of (24) is similar. The (spherically symmetric) symbol for $S(\tau)$ is

$$
S_{\tau}(\lambda)=\frac{\sin \lambda \tau}{\lambda}
$$

To prove the result we use complex interpolation. Consider the analytic family of operators $S_{\tau}^{z}$ with symbols

$$
S_{\tau}^{z}(\lambda)=a(z) S_{\tau}(\lambda)\left(\lambda^{2}+\beta^{2}\right)^{\frac{z}{2}}, \quad \beta>\rho,
$$

where the function $a(z)=e^{z^{2}} \Gamma^{-1}(z+\rho)$ is bounded and holomorphic in the strip $-\rho \leq \Im z \leq 1$. Then by Stein's complex interpolation theorem [18] it suffices to prove that

$$
\left\|S_{\tau}^{z}\right\|_{L^{2} \rightarrow L^{2}} \leq c(1+\tau), \quad \Re z=1
$$

and

$$
\left\|S_{\tau}^{z}\right\|_{L^{1} \rightarrow L^{\infty}} \leq c(\sinh \tau)^{-\rho}, \quad \Re z=-\rho .
$$

The $L^{2}$ estimate (26) follows from the straightforward bound

$$
\left|S_{\tau}^{z}(\lambda)\right| \leq c(1+\tau), \quad \Re z=1 .
$$

Hence, it remains to prove the $L^{1} \rightarrow L^{\infty}$ estimate (27). We need to show that the spherically symmetric kernel $K_{\tau}^{z}(s)$ of the operator $S_{\tau}^{z}$ satisfies the uniform bound

$$
\left|K_{\tau}^{z}(s)\right| \leq c(\sinh \tau)^{-\rho}, \quad \Re z=-\rho .
$$


By (7) the kernel $K_{\tau}^{z}(s)$ can be written in terms of the (spherically symmetric) symbol $S_{\tau}^{z}$ as

$$
\begin{aligned}
K_{\tau}^{z}(s) & =a(z) \int_{0}^{\infty} S_{\tau}^{z}(\lambda) \Phi_{\lambda}(s)|c(\lambda)|^{-2} d \lambda \\
& =a(z) \frac{1}{2} \Re \int_{-\infty}^{\infty} \lambda^{-1} e^{i \lambda \tau}\left(\lambda^{2}+\beta^{2}\right)^{\frac{z}{2}} \Phi_{\lambda}(s) c(\lambda)^{-1} \bar{c}(\bar{\lambda})^{-1} d \lambda .
\end{aligned}
$$

a) Suppose that

$$
\sinh \tau>2 \sinh s .
$$

Then use the Plancherel theorem and (66) to rewrite the integral as

$$
K_{\tau}^{z}(s)=a(z) \Re \frac{2^{\rho-2} \Gamma\left(\rho+\frac{1}{2}\right)}{\Gamma(\rho)(\sinh s)^{2 \rho-1}} \int_{-s}^{s}(\cosh s-\cosh \mu)^{\rho-1} g(\mu-\tau) d \mu,
$$

where $g$ is the Fourier transform of the function

$$
\left(\lambda^{2}+\beta^{2}\right)^{\frac{z}{2}} \lambda^{-1} c(\lambda)^{-1} \bar{c}(\bar{\lambda})^{-1} .
$$

If $\Re z=-\rho$, then by Lemma 2.1 this is a holomorphic $S^{\rho-1}$ symbol inside the strip $|\Im \lambda| \leq \rho$ (with the possible exception of simple poles at $\pm i \rho$ ). Therefore its Fourier transform $g$ satisfies the bound

$$
g(\xi) \leq c|\sinh \xi|^{-\rho}
$$

Hence using (29) we can estimate in (30):

$$
\begin{aligned}
\left|K_{\tau}^{z}(s)\right| & \leq c(\sinh s)^{1-2 \rho}(\cosh s-1)^{\rho-1} \int_{-s}^{s}(\sinh (\mu-\tau))^{-\rho} d \mu \\
& \leq c(\sinh s)^{1-2 \rho}(\cosh s-1)^{\rho-1}\left[(\tanh s)(\sinh (\tau-s))^{-\rho}\right] \\
& =c(\cosh s)^{-1}\left(\cosh \frac{s}{2}\right)^{2-2 \rho}(\sinh (\tau-s))^{-\rho} \\
& \leq c[\cosh s \sinh (\tau-s)]^{-\rho} \\
& \leq c(\sinh \tau)^{-\rho}
\end{aligned}
$$

which gives

$$
\left|K_{\tau}^{z}(s)\right| \leq c(\sinh \tau)^{-\rho}
$$

q.e.d.

b) Suppose now that $2 \sinh s \geq \sinh \tau$, and look for a bound on

$$
K_{\tau}^{z}(s)=\int_{0}^{\infty} \sin \lambda \tau N(\lambda, s) d \lambda
$$

where

$$
N(\lambda, s)=a(z) \lambda^{-1}\left(\lambda^{2}+\beta^{2}\right)^{\frac{z}{2}} \Phi_{\lambda}(s)|c(\lambda)|^{-2} .
$$

Set $z=-\rho+i \sigma$.

b1) The case when $s \geq 1$. Since $c^{-1}(0)=0$, by (13) we obtain for small $\lambda$

$$
|N(\lambda, s)| \leq c(\sinh s)^{-\rho}, \quad|\lambda| \leq 1 .
$$

For large positive $\lambda$ we use the more precise asymptotics in (10), (12) to obtain

$$
N(\lambda, s)=c_{\rho} e^{z^{2}}(\sinh s)^{-\rho}\left(\Gamma^{-1}(i \sigma) \cos \left(\lambda s+\frac{\rho \pi}{2}\right) \lambda^{-1+i \sigma}+O\left(|\lambda|^{-2}\right)\right), \quad|\lambda| \geq 1,
$$


where $c_{\rho}$ is a constant which depends only on $\rho$. Since the Fourier transform of $\Gamma^{-1}(i \sigma) \lambda_{+}^{-1+i \sigma}$ is the bounded function $(\lambda+i 0)^{i \sigma}$, the last two estimates imply the bound

$$
\left|K_{\tau}^{z}(s)\right| \leq c(\sinh s)^{-\rho} .
$$

Since we are in the case $\sinh \tau \leq 2 \sinh s$, the desired conclusion (28) follows. q.e.d.

b2) The case when $s \leq 1$. Then $\tau \leq 2 s$. Since $c^{-1}(0)=0$, by (11) we get the bound

$$
|N(\lambda, s)| \leq c \lambda^{\rho-1}
$$

We can use this bound for $\lambda \leq s^{-1}$ to get

$$
\int_{0}^{s^{-1}}|N(\lambda, s)| d \lambda \leq c s^{-\rho}
$$

On the other hand, for $\lambda \geq s^{-1}$, by (10) and (14) we obtain

$$
N(\lambda, s)=c_{\rho} e^{z^{2}}(\sinh s)^{-\rho}\left(\Gamma^{-1}(i \sigma) \cos \left(\lambda s+\frac{\rho \pi}{2}\right) \lambda^{-1+i \sigma}+O\left(s^{-1}|\lambda|^{-2}\right)\right), \quad \lambda s \geq 1 .
$$

The last two estimates imply the bound

$$
\left|K_{\tau}^{z}(s)\right| \leq(\sinh s)^{-\rho} .
$$

Since we are in the case $\tau \leq 2 s,(28)$ follows. q.e.d.

\section{Global solutions For Semilinear hyperbolic EQUATions}

Consider the semilinear hyperbolic equation

$$
\square u=u^{\alpha} \quad \text { in } \mathbb{R}^{n+1}
$$

with initial data $u(0)=u_{0}, u_{t}(0)=u_{1}$. There are two special values of $\alpha$. One is the conformal exponent,

$$
\alpha_{c}=\frac{n+3}{n-1} .
$$

The other one is $\alpha_{0}$, which is the positive solution of the equation

$$
\rho \alpha(\alpha-1)=\alpha+1 .
$$

Recently Georgiev, Lindblad and Sogge [7] have proved that if $\alpha>\alpha_{0}$ then one has global existence for (33) for small and compactly supported initial data. Their work follows partial results in 11, [13, 21, 12]. It has been known for some time that such a result would be sharp. For $\alpha<\alpha_{0}$, counterexamples were produced in [1] $(n=3)$, 17] (any $n)$.

Our aim here is to provide an alternate and, hopefully, simpler proof of the same result, using the new estimates for the wave equation in the hyperbolic space.

We also consider the critical case $\alpha=\alpha_{0}$, where we prove that "almost global" well-posedness holds, i.e. we prove exponential bounds on the life-span of the solutions. This extends results proved in [14] in dimension $n \leq 8$. However, our bounds are not as good as those in [14]; this reflects the fact that the function spaces we use are Lorentz invariant, i.e. we do not assume any decay at infinity for the initial data in the hyperbolic space. 
Theorem 4. The following hold for solutions to the equation (33) with initial data in the space $H^{1} \times L^{2}$ :

a) Suppose that $\alpha_{c} \geq \alpha>\alpha_{0}$. Then the equation has global solutions for small compactly supported data.

b) Suppose that $\alpha=\alpha_{0}$. Then the lifespan of solutions with compactly supported initial data of size $O(\epsilon)$ is at least $O\left(e^{c \epsilon^{-r}}\right)$, where $r=\frac{(\alpha+1)(\alpha-1)}{3 \alpha+1}$.

From the proof it is clear that one should be able to replace the initial data space with

$$
H^{s+1, p^{\prime}} \times H^{s, p^{\prime}}, \quad p=\alpha p^{\prime}, s=-1+(\rho+1)\left(\frac{1}{p^{\prime}}-\frac{1}{p}\right) .
$$

The technical difficulty is that we want to prove the result for initial data provided on the surface $t=$ const, while in order to do the analysis on the hyperbolic space it is more convenient to assume that the initial data is provided on a hyperboloid $\tau=$ const.

Proof of Theorem 4. Suppose that the initial data at $t=1$ are chosen in the set $2|x|<1$. Then the solution is supported in the forward light cone $t>|x|$. Hence, we can transform the equation into a semilinear hyperbolic equation in the hyperbolic space. Since local well-posedness holds in the energy space $H^{1} \times L^{2}$, without any restriction in generality we can assume that the initial data is provided on the hyperboloid $\tau=0$.

The function $v=e^{\rho \tau} u$ solves the equation

$$
\square_{H} v=e^{\beta \tau} v^{\alpha}, \quad \beta=2+\rho(1-\alpha) .
$$

Note that $\alpha=\alpha_{c}$ corresponds to $\beta=0$ while $\alpha<\alpha_{c}$ corresponds to $\beta>0$.

Choose $p$ so that $p=\alpha p^{\prime}$, i.e. $p=\alpha+1$. If $\alpha \leq \alpha_{c}$ then $s$ in (23) satisfies $1-s \geq s$; therefore (23) implies the estimate

$$
\|S(\tau) f\|_{L^{p}} \leq \frac{(1+\tau)^{\frac{2}{p}}}{(\sinh \tau)^{\rho\left(\frac{1}{p^{\prime}}-\frac{1}{p}\right)}}\|f\|_{L^{p^{\prime}}}
$$

Observe that, using (35), one can easily prove that the solution $v$ to (34) can be extended as long as it remains bounded in $L^{p}$. Hence, in order to estimate its life-span, it suffices to produce appropriate $L^{p}$ bounds.

Using the variation of parameters formula, the estimates (35), (24) yield

$$
\begin{aligned}
\|v(T)\|_{L^{p}} & \leq(1+T)^{\frac{2}{p}}(\sinh T)^{-\gamma}\left(\|v(0)\|_{H^{1, p^{\prime}}}+\left\|v_{\tau}(0)\right\|_{L^{p^{\prime}}}\right) \\
& +\int_{0}^{T} e^{\beta \tau}(\sinh (T-\tau))^{-\gamma}(1+T-\tau)^{\frac{2}{p}}\|v(\tau)\|_{L^{p}}^{\alpha} d \tau
\end{aligned}
$$

where

$$
\gamma=\rho\left(\frac{1}{p^{\prime}}-\frac{1}{p}\right)
$$

Hence, if we set

$$
E(T)=(1+T)^{-\frac{2}{p}} e^{\gamma T}\|v(T)\|_{L^{p}}, \quad\|v(0)\|_{H^{1, p^{\prime}}}+\left\|v_{\tau}(0)\right\|_{L^{p^{\prime}}}=\epsilon,
$$

then for $T>1$ we get

$$
E(T) \leq \epsilon+\int_{0}^{T}(1+\tau)^{\frac{2 \alpha}{p}} e^{(\beta+(1-\alpha) \gamma) \tau}(\tanh (T-\tau))^{-\gamma \tau} E(\tau)^{\alpha} d \tau
$$


Observe that the exponent $\beta+(1-\alpha) \gamma$ equals

$$
\beta+(1-\alpha) \gamma=2+\rho(1-\alpha)\left(2-\frac{2}{p}\right)=2\left(1-\rho \frac{\alpha(\alpha-1)}{\alpha+1}\right),
$$

which is negative if $\alpha>\alpha_{0}$ and 0 if $\alpha=\alpha_{0}$.

Set

$$
T_{\epsilon}=\inf _{E(T) \geq 2 \epsilon} T
$$

Then for $\alpha>\alpha_{0}$ (37) gives

$$
\epsilon \leq c \epsilon^{\alpha}
$$

which is false if $\epsilon$ is sufficiently small. Hence $E(T)<2 \epsilon$ for all $T$, which implies global existence.

If $\alpha=\alpha_{0}$ then $\beta+(1-\alpha) \gamma=0$. Therefore (37) gives

$$
\epsilon \leq\left(1+T_{\epsilon}\right)^{\frac{2 \alpha}{p}+1} \epsilon^{\alpha} .
$$

Hence, for small $\epsilon$ we get

$$
T_{\epsilon} \geq c \epsilon^{\frac{\alpha^{2}-1}{3 \alpha+1}}
$$

But a lifespan of at least $T_{\epsilon}$ in the hyperbolic space implies a lifespan of at least $O\left(e^{T_{\epsilon}}\right)$ in the Minkowski space, and therefore the conclusion follows.

\section{Weighted Strichartz estimates}

The aim of this section is to use the inequality (23) to prove weighted Strichartz type estimates in the hyperbolic space and then to transfer them to the Minkowski space. Thus we recover scale invariant formulations of the estimates used in [7]. Note that, using arguments similar to those in $\mathbb{R}^{n+1}$, one can easily derive the mixed norm space-time Strichartz estimates in the hyperbolic space, which are identical to those in $\mathbb{R}^{n+1}$. However, the estimates with the same $L^{p}$ regularity in all variables can be easily transferred to the Minkowski space.

Our main result is

Theorem 5. Let $u$ be a solution for $\square u=f$ which is supported in the forward light cone. Then the following estimate holds:

$$
\left\|\left(t^{2}-x^{2}\right)^{a} u\right\|_{L^{p}\left(R^{n+1}\right)} \leq\left\|\left(t^{2}-x^{2}\right)^{b} f\right\|_{L^{p^{\prime}}\left(R^{n+1}\right)}
$$

for

$$
2 \leq p \leq \frac{2(n+1)}{n-1}, \quad b<\frac{1}{p}, \quad a=b-1+\frac{n+1}{2}\left(\frac{1}{p^{\prime}}-\frac{1}{p}\right)
$$

The two forbidden endpoints for this estimate are

$$
\left(p=2, a=-\frac{1}{2}, b=\frac{1}{2}\right), \quad\left(p=\frac{2(n+1)}{n-1}, a=b=\frac{1}{p}\right) .
$$

This result follows from (23) combined with the following simple lemma. 
Lemma 6.1. Let $F_{\alpha}$ be the convolution with the kernel $\sinh \tau_{+}^{-\alpha}, 0 \leq \alpha<1$. Then for

$$
1<q \leq p<\infty, \quad \frac{1}{q}-\frac{1}{p} \leq 1-\alpha, \quad \beta<\alpha,
$$

we have

$$
\left\|e^{\beta \tau} F_{\alpha} f\right\|_{L^{p}(\mathbb{R})} \leq\left\|e^{\beta \tau} f\right\|_{L^{q}(\mathbb{R})}
$$

Proof of Lemma 6.1. The translation invariant kernel of the operator

$$
F_{\alpha, \beta}=e^{\beta \tau} F_{\alpha} e^{-\beta \tau}
$$

is

$$
K(\tau)=e^{\beta \tau}\left(\sinh \tau_{+}\right)^{-\alpha} .
$$

This is both integrable and bounded by $|\tau|^{-\alpha}$, and so the conclusion follows from the Hardy-Littlewood-Sobolev inequality.

Proof of Theorem 5. The forward solutions $v$ to $\square_{H} v=g$ in $\mathbb{R} \times \mathbb{H}^{n}$ are represented as

$$
v(\tau)=\int_{-\infty}^{\tau} S(\tau-\tilde{\tau}) g(\tilde{\tau}) d \tilde{\tau}
$$

Then we can combine the above lemma with (35) to get

$$
\left\|e^{\beta \tau} v\right\|_{L^{p}\left(\mathbb{R} \times \mathbb{H}^{n}\right)} \leq\left\|e^{\beta \tau} g\right\|_{L^{p^{\prime}}\left(\mathbb{R} \times \mathbb{H}^{n}\right)}
$$

for

$$
\beta<\rho\left(\frac{1}{p^{\prime}}-\frac{1}{p}\right), \quad 2 \leq p \leq \frac{2(n+1)}{n-1} .
$$

Set $v=e^{\rho \tau} u$. Then $v$ solves $\square_{H} v=g$, with $g=e^{(\rho+2) \tau} f$. Hence, applying (41) to $v$ and changing coordinates, we get

$$
\left\|e^{\left(\beta-\frac{n+1}{p}+\rho\right) \tau} u\right\|_{L^{p}\left(\mathbb{R}^{n+1}\right)} \leq\left\|e^{\left(\beta-\frac{n+1}{p^{\prime}}+\rho+2\right) \tau} f\right\|_{L^{p^{\prime}}\left(\mathbb{R}^{n+1}\right)} .
$$

Since $e^{2 \tau}=t^{2}-x^{2}$, the conclusion follows.

\section{REFERENCES}

[1] J. Bergh and J. Löfström, Interpolation Spaces: An Introduction, Springer-Verlag, 1976 MR 58:2349

[2] W.O. Bray, Aspects of Harmonic Analysis on Real Hyperbolic Space Functional Analysis, (Orono, ME, 1992), Lecture Notes in Pure and Applied Mathematics 157, Marcel Dekker, 1994, pp. 77-102. MR 95k:43008

[3] P. Brenner, On $L_{p}-L_{p}^{\prime}$ estimates for the wave equations, Math. Z. 145(1975), 251-254 MR 52:8658

[4] J. Clerc and E.M. Stein, $L^{p}$ multipliers for noncompact symmetric spaces, Proc. Nat. Acad. Sci. USA 71(1974), 3911-3912 MR 51:3803

[5] R. Glassey, Existence in the large for $\square u=F(u)$ in two space dimensions, Math. Z. 178(1981), 233-261 MR 84h:35106

[6] J. Ginibre and G. Velo, Generalized Strichartz inequality for the wave equation, J. Funct. Anal. 133 (1995), 50-68. [MR 97a:46047]

[7] V. Georgiev, H. Lindblad and C. Sogge, Weighted Strichartz estimates and global existence for semilinear wave equations, Amer. J. Math. 119 (1997), 1291-1319 MR 99f:35134 
[8] M. Grillakis, Regularity and asymptotic behavior of the wave equation with a critical nonlinearity, Ann. of Math. 132(1990), 485-509 [MR 92c:35080

[9] M. Grillakis, Regularity for the wave equation with a critical nonlinearity, Comm. Pure Appl. Math 45 (1992) 749-774 MR 93e:35073

[10] S. Helgason, Groups and Geometric Analysis, Academic Press 1984 MR 86c:22017

[11] F. John, Blow-up of solutions of nonlinear wave equations in three space dimensions, Manuscripta Math 28 (1979), 235-265 MR 80i:35114

[12] H. Kubo, On the critical decay and power for semilinear wave equations in odd space dimensions, Discrete Contin. Dynam. Systems 2 (1996), 173-190 MR 96m:35221

[13] H. Lindblad and C. Sogge, On existence and scattering with minimal regularity for semilinear wave equations, J Funct. Anal., 1995, 357-426 MR 96i:35087

[14] H. Lindblad and C. Sogge, Long-time existence for small amplitude semilinear wave equation, Amer. J. Math. 118 (1996), 1047-1135 MR 97h:35158

[15] H. Pecher Non-linear small data scattering for the wave and Klein-Gordon equations, Math. Z. 185(1984), 261-270 MR 85h:35165

[16] I. Segal, Space-time decay for solutions of wave equations, Adv. in Math. 22(1976), 305-311 MR 58:11945

[17] T. Sideris, Nonexistence of global solutions to semilinear wave equations in high dimensions, Comm. PDE 12 (1987), 378-406 MR 86d:35090

[18] E.M. Stein, Interpolation of linear operators, Trans. Amer. Math. Soc. 83 (1956), 482-492 MR 18:575d

[19] E.M. Stein, Harmonic Analysis, Princeton University Press, 1993 MR 95c:42002

[20] R.S. Strichartz, Restrictions of Fourier transform to quadratic surfaces and decay of solutions of Wave Equations, Duke Math. J. 44(1977) 705-714 MR 58:23577

[21] Y. Zhou, Cauchy problem for semilinear wave equations in four space dimensions with small initial data, J. Partial Diff. Equations 8 (1995) 135-144 MR 96c:35128

Department of Mathematics, Princeton University, Princeton, New Jersey 08540 Current address: Department of Mathematics, Northwestern University, Evanston, Illinois 08540

E-mail address: tataru@math.nwu.edu 